\title{
Pembrolizumab weight based dosing - A call for policy change
}

\author{
Amol Patel ${ }^{1 \odot}$ Amol Akhade ${ }^{2}$ Purvish Parikh ${ }^{3} \quad$ Atul Sharma $^{4}$ Hemant Malhotra ${ }^{5}$ \\ Kumar Prabhash ${ }^{6}$ Govind Babu ${ }^{7}$ (i) Vanita Noronha ${ }^{6}$ Ullas Batra ${ }^{8}$ Prashant Mehta ${ }^{9}$ \\ Vineet Govinda Gupta ${ }^{10}$ Venkatraman Radhakrishnan ${ }^{11}$ Rakesh Reddy Boya ${ }^{12}$ Bivas Biswas ${ }^{13}$
}

${ }^{1}$ Department of Medicine, Oncology Centre, INHS, ASVINI, Mumbai

${ }^{2}$ Department of Medical Oncology, Topiwala National Medical College and Nair Hospital, Mumbai, Maharashtra, India

${ }^{3}$ Department of Medical Oncology, Mumbai Oncocare Centers, Mumbai, Maharashtra, India

${ }^{4}$ Department of Medical Oncology, AlIMS, New Delhi, India

${ }^{5}$ Department of Medical Oncology, Mahatmma Gandhi Medical College Hospital, Jaipur, Rajasthan, India

${ }^{6}$ Department of Medical Oncology, Tata Memorial Hospital, Mumbai, Maharashtra, India

${ }^{7}$ Department of Medical Oncology, HCG and St John's Medical College Hospital, Bengaluru, Karnataka, India

8 Department of Medical Oncology, Rajiv Gandhi Cancer Institute and Research Centre, Delhi, India

Ind ] Med Paediatr Oncol 2022;43:306-310.
Address for correspondence Amol Akhade, DM, Department of Medical Oncology, Topiwala National Medical College and Nair Hospital, Mumbai, Maharashtra 400008, India

(e-mail: amolakhade@yahoo.co.in).

${ }^{9}$ Department of Medical Oncology, Asian Institute of Medical Sciences, Faridabad, Haryana, India

10 Department of Medical Oncology, Artemis Hospital, Gurugram, Haryana, India

${ }^{11}$ Department of Medical Oncology, Cancer Institute, Chennai, Tami Nadu, India

${ }^{12}$ Department of Medical Oncology, Mahatma Gandhi Cancer Hospital, Vishakhapatnam, Andhra Pradesh, India

${ }^{13}$ Department of Medical Oncology, Tata Medical Center, Kolkata, West Bengal, India

\section{Introduction}

Immuno-oncology (IO) drugs are now approved for use in metastatic, adjuvant, and/or neoadjuvant setting for a growing list of cancers including non-small cell lung cancer, small cell lung cancer, melanoma, urothelial cancer, renal cell carcinoma, head and neck squamous cell carcinoma, esophageal cancer, gastric cancer, cervical cancer, endometrial cancer, hepatocellular cancer, Merkel cell carcinoma, microsatellite instability-high or mismatch repair deficient colorectal cancer and other cancers, tumor mutational burdenhigh cancer, cutaneous squamous cell carcinoma, triplenegative breast cancer, classical Hodgkin lymphoma, and primary mediastinal large B cell lymphoma. This list continues to expand at an impressive speed. The benefits in improving progression-free survival (PFS) and overall survival are a welcome advance beyond the plateau that we seem to have reached with conventional chemotherapy treatment. The biggest challenge is to identify biomarkers that would help select the 20 to $40 \%$ patients who respond to immunotherapy drugs, thereby limiting treatment to those

most likely to benefit, avoiding toxicity among the patients unlikely to respond. This has very important pharmacoeconomic implications, especially because immunotherapy drugs are very expensive.

The important question is whether we can do something substantial to address unnecessary wastage of expensive medicines. The answer is yes. It is based on a long established principle of using dose according to the individual's weight/surface area. It is based on avoiding "flat" dosing-a flawed strategy that leads to unnecessary wastage of expensive medicines and subjects our patients to avoidable toxicity. There is a strong rationale to integrate the concepts of pharmacoeconomics. ${ }^{1}$ We present here our policy brief on rational use of pembrolizumab that optimizes patient benefit as well as utilization of resources.

\section{Pharmacokinetics and Pharmacodynamics of Pembrolizumab}

Pembrolizumab is a completely humanized monoclonal antibody immunoglobulin G 4 against the programmed

DOI https://doi.org/ $10.1055 / \mathrm{s}-0042-1742651$. ISSN 0971-5851. (c) 2022. Indian Society of Medical and Paediatric Oncology. All rights reserved.

This is an open access article published by Thieme under the terms of the Creative Commons Attribution-NonDerivative-NonCommercial-License, permitting copying and reproduction so long as the original work is given appropriate credit. Contents may not be used for commercial purposes, or adapted, remixed, transformed or built upon. (https://creativecommons.org/ licenses/by-nc-nd/4.0/)

Thieme Medical and Scientific Publishers Pvt. Ltd., A-12, 2nd Floor, Sector 2, Noida-201301 UP, India 
death-1 tumor-derived antigen. Similar to other monoclonal antibodies, it has peculiar pharmacokinetics and pharmacodynamics (PK PD) properties such as a very long half-life, linear clearance at doses above $0.3 \mathrm{mg} / \mathrm{kg}$, and steady-state levels being achieved after 18 weeks of therapy. ${ }^{2}$ Using the half-life of 23.72 days for pembrolizumab, the complete washout period will be 237 days. ${ }^{3}$ Washout period is generally 10 half-lives. ${ }^{4}$ In phase 1 pharmacokinetic study of pembrolizumab, the complete interleukin 2 target engagement was seen at doses of $1 \mathrm{mg} / \mathrm{kg}$ and above. ${ }^{5}$ Durability of this action was documented till day 21 in all the three-dose cohorts, that is, 1,3 , or $10 \mathrm{mg} / \mathrm{kg}$ and responses lasted beyond stopping the drug. ${ }^{5}$ The modeling-based studies by Chatterjee et al in melanoma and metastatic non-small cell lung cancer (mNSCLC) provided additional evidence that $2 \mathrm{mg} / \mathrm{kg}$ q3 weeks was the optimal dose. ${ }^{6,7}$ The use of pembrolizumab beyond $2 \mathrm{mg} / \mathrm{kg}$ q3 weeks has not shown to improve response rates. Safety, adverse effects, and activity of pembrolizumab were studied at $2 \mathrm{mg} / \mathrm{kg}$ every 2 weeks, $10 \mathrm{mg} / \mathrm{kg}$ every 2 weeks, and $10 \mathrm{mg} / \mathrm{kg}$ every 3 weeks in advanced or mNSCLC. $^{8}$ It is important to note that, in this study, the response rate was highest for the dose of $2 \mathrm{mg} / \mathrm{kg}$ every 2 weeks (33.3\%; albeit with limited data from 6 patients). Also, it was identical for pembrolizumab $10 \mathrm{mg} / \mathrm{kg}$ q3 weeks $(19.2 \% ; n=287)$ and $10 \mathrm{mg} / \mathrm{kg}$ q2 weeks $(19.3 \%$; $n=202$ ) schedules, irrespective of histology.

The phase 2/3 KEYNOTE-010 study enrolled 1,034 patients of previously treated mNSCLC across 24 countries. Pembrolizumab 2 and $10 \mathrm{mg} / \mathrm{kg}$ (doses selected on the basis of the previous studies-vide supra) was compared with docetaxel. Overall survival was the primary end point, being 10.4, 12.7, and 8.4 months in pembrolizumab $2 \mathrm{mg} / \mathrm{kg}, 10 \mathrm{mg} / \mathrm{kg}$, and docetaxel groups, respectively. Hazard ratios (HRs) were 0.71 ( $2 \mathrm{mg} / \mathrm{kg} \mathrm{q3w}$ ) and 0.61 (10 mg/kg q3w) in comparison with docetaxel. In patients with $50 \%$ and more programmed death ligand 1 (PD-L1) expression, HRs were 0.54 and 0.50 for overall survival in pembrolizumab 2 and $10 \mathrm{mg} / \mathrm{kg}$ groups, respectively. The authors therefore had sufficient evidence to support the dose of $2 \mathrm{mg} / \mathrm{kg}$ q3 weeks for pembrolizumab. ${ }^{9}$

Similarly, a phase 2 randomized trial in ipilimumab refractory metastatic melanoma studied pembrolizumab at 2 and $10 \mathrm{mg} / \mathrm{kg}$ every 3 weeks. The HR for PFS was 0.57 and 0.50 in 2 and $10 \mathrm{mg} / \mathrm{kg}$ dose and 6-month PFS rate was 34 and $38 \%$, respectively. The authors recommended the minimal effective dose of $2 \mathrm{mg} / \mathrm{kg}$ q3 weeks for further use, although the trial was not designed to compare outcome between the two dose schedules. ${ }^{10}$

Factors known to affect the metabolism of pembrolizumab include age, sex, serum albumin, and performance status. $^{2}$ Turner et al's PK PD study concluded that patients with slow clearance have almost double the overall survival as compared with those who had rapid clearance, with identical benefit in cohorts of 2 and $10 \mathrm{mg} / \mathrm{kg}$ dose. ${ }^{11}$ With all this evidence, $2 \mathrm{mg} / \mathrm{kg}$ every 3 weeks is an optimal dose.

\section{Standard of Care and Approved Dosing}

In the landmark KEYNOTE-024 trial of pembrolizumab in advanced lung cancer, ${ }^{12}$ the flat dose of $200 \mathrm{mg}$ was used every 3 weeks, citing pharmacokinetic modeling by Freshwater et al. Freshwater et al used data from population-based pharmacokinetic properties based on 2 and $10 \mathrm{mg} / \mathrm{kg}$ and compared them to data from newer trials using fixed doses. In fact, they found no difference between weight based and flat dose regimens. ${ }^{13}$

Food and Drug Administration approved pembrolizumab $400 \mathrm{mg}$ dose every 6 weeks across all indications. This approval was based on pharmacokinetic modeling study which compared 6 weekly $400 \mathrm{mg}$ schedule to $200 \mathrm{mg} 3$ weekly and $2 \mathrm{mg} / \mathrm{kg} 3$ weekly schedules and concluded that the three schedules were expected to produce similar efficacy and safety across all diseases. ${ }^{14}$ This work was supported by Merck Sharp \& Dohme Corp. The authors specified that a fixed dose of $200 \mathrm{mg}$ q3 weeks would be more convenient by reducing cost in respect of logistics (reducing cost of manufacturer and hospitals). They conveniently missed the adverse implication on payors (patients, insurance companies, government) by shutting out the weight-based schedule of $2 \mathrm{mg} / \mathrm{kg}$. Further phase 3 trials continued to propagate flat doses of $200 \mathrm{mg}$ across various cancer groups-without any efficacy, safety, or cost-benefit rationale.

The present standard of care for dose differs with different indications. Based on the patent holding company's dossier submission to Indian drug regulatory authorities (Drug Controller General of India), pembrolizumab has been approved since 2016 for use in lung cancer and melanoma at the dose of $2 \mathrm{mg} / \mathrm{kg}$ q3 weeks (in previously treated patients of mNSCLC and melanoma). In contrast, for treatment naïve patients of MNSCLC, the submission dossier recommended a flat dose of $200 \mathrm{mg}$ q3 weeks. $^{15}$ National Comprehensive Cancer Network recommended $2 \mathrm{mg} / \mathrm{kg}$ every 3 weeks for malignant melanoma in addition to flat dose of $200 \mathrm{mg}^{16}$

\section{Weight-Based versus Flat Dosing of Pembrolizumab}

The flat dose of $200 \mathrm{mg}$ every 3 weeks was derived from the modeling-based study. ${ }^{13}$ The Canadian Agency for Drugs and Technologies in Health (CADTH) extensively reviewed the pharmacokinetics and modeling-based studies of pembrolizumab and nivolumab ${ }^{3}$ and derived that $2 \mathrm{mg} / \mathrm{kg}$ every 3 weeks is the optimal dose of pembrolizumab. In CADTH report, blood levels of pembrolizumab for a patient of $70 \mathrm{~kg}$ patient were identical between the four cohorts viz. $98 \%$ at the dose of $2 \mathrm{mg} / \mathrm{kg}$ q3 weeks; $97 \%$ for $4 \mathrm{mg} / \mathrm{kg}$ q6h weeks dosing; $99.31 \%$ at $200 \mathrm{mg}$ q3 weeks; and $98.16 \%$ for $400 \mathrm{mg}$ q6 weeks. ${ }^{3}$

Studies have shown that pembrolizumab is well tolerated up to doses of $10 \mathrm{mg} / \mathrm{kg}$ q2 weeks. Toxicities are similar between weight-based ( $2 \mathrm{mg} / \mathrm{kg}$ q3 weeks) and fixed-dose (200 mg q3 weeks) schedules. Recent meta-analysis showed that $2 \mathrm{mg} / \mathrm{kg}$ dose of pembrolizumab was better tolerated. Interestingly, in this meta-analysis, pembrolizumab discontinuation rate was higher $(9.2 \%$; $95 \% \mathrm{Cl}: 6.9-12 \%)$ in flat $200 \mathrm{mg}$ dose as compared with weight-based dosing schedule (6.5\%; $95 \%$ CI: $4.8-8.8 \%) .{ }^{17}$ 


\section{Real-World Studies}

Mukherjee et al studied the flat dose versus weight-based dose of pembrolizumab and nivolumab. A total of 60/137 patients received weight-based dosing. There was no difference in overall survival, even after adjusting for other study variables. ${ }^{18}$ In a similar study from Singapore, $100 \mathrm{mg}$ (low dose) of pembrolizumab was compared with $200 \mathrm{mg}$ dose in mNSCLC. Sixty-five patients received $100 \mathrm{mg}$ pembrolizumab and 49 patients received $200 \mathrm{mg}$ flat dose. Again, there was no difference in response rate, PFS and overall survival. $^{19}$

Goldstein et al analyzed the cost implications for United States if pembrolizumab is used with weight-based $2 \mathrm{mg} / \mathrm{kg}$ dose in PD-L1-positive lung cancer treatment. They estimated savings of $\$ 82.5$ million every year without compromising patient outcomes in any way. ${ }^{20}$ Using this approach for all labeled indications of pembrolizumab would multiply this saving many folds, saving billions of dollars every year in the United States alone. Another publication also supported the weight-based $4 \mathrm{mg} / \mathrm{kg}$ every 6 weeks schedule during coronavirus disease 2019 (COVID-19) pandemic, with additional benefits. It would deliver similar results (efficacy and safety), save cost, and reduce the risk of COVID-19 exposure by reducing hospital visits. ${ }^{21}$

\section{Worldwide Challenges and Solutions}

The 23rd expert committee of World Health Organization on use of essential medicines has considered cost as a major factor for nonconsidering the application for pembrolizumab as essential medicine and also stated that $\mathrm{mg} / \mathrm{kg}$ dosing may be preferred to flat dose without compromising on disease outcomes. ${ }^{22}$ CADTH is an independent organization which helps the Canadian authorities by providing objective evidence on optimal use of dose, devices, and procedures. ${ }^{3}$ It has recommended use of pembrolizumab as $2 \mathrm{mg} / \mathrm{kg}$ (with $200 \mathrm{mg}$ capping dose) for first line in NSCLC and melanoma. Vial sharing option was suggested specifically as the $50 \mathrm{mg}$ vial was not available. Recently, Israel and Denmark approved weight-based dosing of pembrolizumab.

\section{Access to Immuno-oncology Drug in Indian Setting}

In a real-world study of more than 9,000 patients, only $1.61 \%$ of eligible patients received immunotherapy from 2015 to 2018. The cost remains a major deterrent for access to costly medicines. ${ }^{23}$ Average weight of Indian lung cancer patients is $55 \mathrm{~kg}$ as compare with $75 \mathrm{~kg}$ of average American patients. ${ }^{24,25}$ The average weight of Indian male is $59.6 \mathrm{~kg}$ and female is $52.5 \mathrm{~kg} .{ }^{26}$ In studies from north Indian lung cancer patients, almost $45 \%$ of patients were underweight (body mass index $<18.49 \mathrm{~kg} / \mathrm{m}^{2}$ and at 70 percentiles, weight was less than $60 \mathrm{~kg}$ ).

The cost of pembrolizumab is around INR 235,000 per $100 \mathrm{mg}$ vial (with one vial free). This price might differ marginally across various states and institutions. Cost of $100 \mathrm{mg}$, that is, one vial of pembrolizumab is more than India's per capita gross domestic product. A $100-\mathrm{mg}$ vial is ideal for a patient weighing $50 \mathrm{~kg}$, saving a vial for a vial. If conventional doses are used, there is huge monetary loss to the payers. For patients, more than $60 \mathrm{~kg}$, vial sharing remains an option and same vial can be used multiple times as drug is available in liquid form.

\section{Practical Challenges and Remedies for Practicing the $\mathbf{m g} / \mathbf{k g}$ Dosing}

\section{The Dose Banding}

Dose banding is a well-established methodology wherein the weight-based calculations are simplified by rounding off, taking into consideration available vial size/strength. Ogungbenro et al have shown how this strategy in the usage of pembrolizumab and nivolumab is cost effective. ${ }^{27}$ In fact, the National Health Service of the United Kingdom has adopted the strategy of dose banding for nivolumab and pembrolizumab. ${ }^{28,29}$ They round off to allow $10 \%$ variation. Thus, for a typical 45 to $55 \mathrm{~kg}$ patient, the dose range would be 90 to $110 \mathrm{mg}$ of pembrolizumab and one vial of $100 \mathrm{mg}$ would be sufficient, and for majority of Indian patients, the cost of treatment will be halved.

\section{Stability of 10 Drugs}

Stability has been studied at 2 to $8^{\circ} \mathrm{C}$, at $40^{\circ} \mathrm{C}$, and using freezing/thawing cycles. This has been documented for both 10 and $2 \mathrm{mg} / \mathrm{mL}$ concentrations. Pembrolizumab is stable in normal saline $0.9 \%$ at 2 to $8^{\circ} \mathrm{C}$ for up to 7 to 14 days in polyolefin infusion bags. ${ }^{30,31}$ Nivolumab is stable at 2 to $8^{\circ} \mathrm{C}$ for 30 days and at $40^{\circ} \mathrm{C}$ for 7 days. $^{32}$

\section{The Implications of the 50-mg Vial}

Key advantage of weight-based dosing is dependent on the availability of a $50-\mathrm{mg}$ vial. Curiously, the $50 \mathrm{mg}$ vial was withdrawn from the U.S. market, while continuing to be available in certain European countries. In the absence of the $50 \mathrm{mg}$ vial, the application (and resultant cost saving) of weight-based dosing disappears in most countries. It would still be possible (but shall increase complexity) in countries that permit vial sharing, where a central pharmacy prepares chemotherapy infusions. Weight-based dosing is the only way of using pembrolizumab in the pediatric populationapproved dose being $2 \mathrm{mg} / \mathrm{kg}$ q3 weeks. ${ }^{33}$ This is applicable to several cancers including classical Hodgkin lymphoma, primary mediastinal B cell lymphoma, metastatic Merkel cell carcinoma, and high-level microsatellite instability tumors. ${ }^{34}$ For a $25-\mathrm{kg}$ child, the dose of $50 \mathrm{mg}$ is adequate. The nonavailability of the $50 \mathrm{mg}$ vial in countries where pediatric use is licensed results in wastage of up to half the drug.

\section{Recommendations by the Authors}

1. Pembrolizumab is recommended as $2 \mathrm{mg} / \mathrm{kg}$ every 3 weekly or $4 \mathrm{mg} / \mathrm{kg}$ every 6 weekly across all indications 
in cost constraint situations, and $200 \mathrm{mg}$ every 3 weeks schedule remains an option.

2. The $50 \mathrm{mg}$ vial of pembrolizumab should be made available in all countries to reduce cost and prevent wastage.

3. Sharing of vials will provide additional saving and should be used where feasible, especially when the government is the payor.

\section{Conclusion}

The usage of IO drugs is expected to rise exponentially in coming years. We provide existing evidence and rationale for usage of pembrolizumab in a manner that optimizes benefit, minimizes toxicity, and prevents unnecessary wastage of resources. These recommendations needs to be implemented and governing authorities are requested to take the case forward.

\section{Methodology}

We initially surveyed the challenges associated with practice of $\mathrm{mg} / \mathrm{kg}$ dosing of pembrolizumab. We discussed this issue with imminent and various medical oncologists of India. We formulated a group of 14 medical oncologists representing nationwide academic and nonacademic institutes, corporate and government hospitals. First author wrote the initial manuscript and the manuscript was circulated to all members of the group through emails. The consensus was accepted and approved unanimously.

\section{Conflict of Interest}

None declared.

\section{References}

1 Tannock IF, Patel A. Pharmacoeconomics. Ind J Med Paediatr Oncol 2021;42:218-220

2 Centanni M, Moes DJAR, Trocóniz IF, Ciccolini J, van Hasselt JGC. Clinical pharmacokinetics and pharmacodynamics of immune checkpoint inhibitors. Clin Pharmacokinet 2019;58(07): 835-857

3 Canadian Agency for Drugs and Technologies in Health. CADTH technology review: optimal use 360 report: dosing and timing of immuno-oncology drugs. Published online November 2019. Accessed May 19, 2020 at: https://www.cadth.ca/sites/ default/ files/ou-tr/ho0008-dosing-timing- immuno-oncology-drugs.pdf

4 Health Canada. Guidance Document: Conduct and Analysis of Comparative Bioavailability Studies [Internet], 2012. Accessed August 29, 2021 at: https://www.canada.ca/en/health-canada/services/drugs-health-products/drug-products/applicationssubmissions/guidance-documents/bioavailability-bioequivalence/conduct-analysis-comparative.html

5 Patnaik A, Kang SP, Rasco D, et al. Phase I study of pembrolizumab (MK-3475; anti-PD-1 monoclonal antibody) in patients with advanced solid tumors. Clin Cancer Res 2015;21(19): 4286-4293

6 Chatterjee M, Turner DC, Felip E, et al. Systematic evaluation of pembrolizumab dosing in patients with advanced non-small-cell lung cancer. Ann Oncol 2016;27(07):1291-1298

7 Chatterjee MS, Elassaiss-Schaap J, Lindauer A, et al. Population pharmacokinetic/pharmacodynamic modeling of tumor size dy- namics in pembrolizumab-treated advanced melanoma. CPT Pharmacometrics Syst Pharmacol 2017;6(01):29-39

8 Garon EB, Rizvi NA, Hui R, et al; KEYNOTE-001 Investigators. Pembrolizumab for the treatment of non-small-cell lung cancer. $\mathrm{N}$ Engl J Med 2015;372(21):2018-2028

9 Herbst RS, Baas P, Kim D-W, et al. Pembrolizumab versus docetaxel for previously treated, PD-L1-positive, advanced non-small-cell lung cancer (KEYNOTE-010): a randomised controlled trial. Lancet 2016;387(10027):1540-1550

10 Ribas A, Puzanov I, Dummer R, et al. Pembrolizumab versus investigator-choice chemotherapy for ipilimumab-refractory melanoma (KEYNOTE-002): a randomised, controlled, phase 2 trial. Lancet Oncol 2015;16(08):908-918

11 Turner DC, Kondic AG, Anderson KM, et al. Pembrolizumab exposure-response assessments challenged by association of cancer cachexia and catabolic clearance. Clin Cancer Res 2018; 24(23):5841-5849

12 Reck M, Rodríguez-Abreu D, Robinson AG, et al; KEYNOTE-024 Investigators. Pembrolizumab versus chemotherapy for PD-L1positive non-small-cell lung cancer. N Engl J Med 2016;375(19): 1823-1833

13 Freshwater T, Kondic A, Ahamadi M, et al. Evaluation of dosing strategy for pembrolizumab for oncology indications. J Immunother Cancer 2017;5:43

14 Lala M, Li TR, de Alwis DP, et al. A six-weekly dosing schedule for pembrolizumab in patients with cancer based on evaluation using modelling and simulation. Eur J Cancer 2020;131:68-75

15 Keytruda prescribing information (package insert is based on worldwide physician circular S-CCDS-MK3475-IV-112017). 2017

16 National Comprehensive Cancer Network guidelines on cutaneous melanoma. Accessed October 10, 2021 at: https://www.nccn. org/professionals/physician_gls/pdf/cutaneous_melanoma.pdf

17 Patail NK, Sher AF, Wu S. Improving tolerability of pembrolizumab with weight based dosing: a meta-analysis. Journal of Clinical Oncology 2021;39(15):2639-2639

18 Mukherjee S, Ibrahimi S, Machiorlatti M, et al. Personalized dosing versus fixed dosing of immune checkpoint inhibitors: a cost analysis study. Am J Ther 2018;25(06):e767-e768

19 Low JL, Huang Y, Sooi K, et al. Low-dose pembrolizumab in the treatment of advanced non-small cell lung cancer. Int J Cancer 2021;149(01):169-176

20 Goldstein DA, Gordon N, Davidescu M, et al. A phamacoeconomic analysis of personalized dosing vs fixed dosing of pembrolizumab in firstline PD-L1-positive non-small cell lung cancer. J Natl Cancer Inst 2017;109(11)

21 Goldstein DA, Ratain MJ, Saltz LB. Weight-based dosing of pembrolizumab every 6 weeks in the time of COVID-19. JAMA Oncol 2020;6(11):1694-1695

22 23rd Expert Committee on Selection and Use of Essential Medicines [Internet]. Accessed September 26, 2021 at: https://www.who. int/news-room/events/detail/2021/06/21/default-calendar/23rdexpert-committee-on-selection-and-use-of-essential-medicines

23 Noronha V, Abraham G, Patil V, et al. A real-world data of immune checkpoint inhibitors in solid tumors from India. Cancer Med 2021;10(05):1525-1534

24 Singh N, Aggarwal AN, Gupta D, Behera D. Prevalence of low body mass index among newly diagnosed lung cancer patients in North India and its association with smoking status. Thorac Cancer 2011;2(01):27-31

25 Kaur H, Sehgal IS, Bal A, et al. Evolving epidemiology of lung cancer in India: reducing non-small cell lung cancer-not otherwise specified and quantifying tobacco smoke exposure are the key. Indian J Cancer 2017;54(01):285-290

26 Average height of men and women worldwide [Internet]. Worlddata.info. Accessed November 23, 2021 at: https://www. worlddata.info/average-bodyheight.php

27 Ogungbenro K, Patel A, Duncombe R, Nuttall R, Clark J, Lorigan P. Dose rationalization of pembrolizumab and nivolumab using 
310 Pembrolizumab weight-based dosing - A call for policy change Patel et al.

pharmacokinetic modeling and simulation and cost analysis. Clin Pharmacol Ther 2018;103(04):582-590

28 National dose banding table for pembrolizumab, NHS England. Accessed January 1, 2022 at: https://www.england.nhs.uk/publication/national-dose-banding-table-pembrolizumab-25-mgml/

29 National dose banding table for nivolumab, NHS England. Accessed January 1, 2022 at: https://www.england.nhs.uk/ publication/national-dose-banding-table-nivolumab-10mgml/

30 Piccoli M, Selmin F, Mangoni G, et al. 3PC-044 In use physicochemical stability of pembrolizumab under the dilution condition required for use in a day hospital. European Journal of Hospital Pharmacy 2020;27(Suppl 1):A42-A42

31 Sundaramurthi P, Chadwick S, Narasimhan C. Physicochemical stability of pembrolizumab admixture solution in normal saline intravenous infusion bag. J Oncol Pharm Pract 2020;26(03): 641-646

32 Le Guyader G, Vieillard V, Mouraud S, Do B, Marabelle A, Paul M. Stability of nivolumab in its original vials after opening and handing in normal saline bag for intravenous infusion. Eur J Cancer 2020;135:192-202

33 Geoerger B, Kang HJ, Yalon-Oren M, et al. Pembrolizumab in paediatric patients with advanced melanoma or a PD-L1-positive, advanced, relapsed, or refractory solid tumour or lymphoma (KEYNOTE-051): interim analysis of an open-label, single-arm, phase 1-2 trial. Lancet Oncol 2020;21(01):121-133

34 Highlights of prescribing information. Accessed January 1,2022 at: https://www.accessdata.fda.gov/drugsatfda_docs/label/2020/ 125514s066lbl.pdf 